\title{
The effects of chemical, biological and physical aging and soil addition on sorption of pyrene to activated carbon and biochar
}

\section{Sarah E Hale*1, Kelly Hanley², Johannes Lehmann², Andrew R Zimmerman $^{3}$ and Gerard Cornelissen ${ }^{1,4,5}$}

1) Department of Environmental Engineering, Norwegian Geotechnical Institute (NGI), P.O. Box 3930, Ullevål Stadion, N-0806, Oslo, Norway

2) Department of Crop and Soil Sciences, Cornell University, Ithaca, NY 14853, USA

3) Department of Geological Sciences, University of Florida, 241 Williamson Hall, P.O.

Box 112120, Gainesville, FL, 32611

4) Department of Applied Environmental Sciences, Stockholm University, 10691 Stockholm, Sweden

5) Institute for Plant and Environmental Sciences, University of Life Sciences, 5003 Ås, Norway

*corresponding author: 00472202 3092, email: sarah.hale@ngi.no

Supporting Information

Table 1: Concentrations of $\mathrm{Ca}, \mathrm{K}, \mathrm{Na}$ and $\mathrm{Mg}$ in the $\mathrm{BC}$ alone and mixed with soil

\begin{tabular}{llllll}
\hline Material & $\begin{array}{l}\text { Aging } \\
\text { regime }\end{array}$ & $\begin{array}{l}\mathrm{Ca} \\
\mathrm{cmolc} / \mathrm{kg}\end{array}$ & $\begin{array}{l}\mathrm{K} \\
\mathrm{cmolc} / \mathrm{kg}\end{array}$ & $\begin{array}{l}\mathrm{Na} \\
\mathrm{cmolc} / \mathrm{kg}\end{array}$ & $\begin{array}{l}\mathrm{Mg} \\
\mathrm{cmolc} / \mathrm{kg}\end{array}$ \\
\hline $\mathrm{AC}$ & UNAGED & 70 & 3 & 1 & 15
\end{tabular}




\begin{tabular}{|c|c|c|c|c|c|}
\hline & $\mathrm{BIO}$ & 62 & 14 & 0.9 & 13 \\
\hline & CHEM 60 & 65 & nd & 2 & 15 \\
\hline & CHEM & 64 & nd & 3 & 17 \\
\hline & 110 & & & & \\
\hline & PHYS & 67 & 8 & 1 & 15 \\
\hline \multirow[t]{6}{*}{ Biochar } & UNAGED & 97 & 413 & 7 & 63 \\
\hline & $\mathrm{BIO}$ & 80 & 390 & 7 & 55 \\
\hline & CHEM 60 & 97 & 428 & 7 & 43 \\
\hline & CHEM & 82 & 460 & 9 & 57 \\
\hline & 110 & & & & \\
\hline & PHYS & 85 & 375 & 6 & 47 \\
\hline \multirow[t]{6}{*}{ AC+soil } & UNAGED & 122 & nd & 0.04 & 21 \\
\hline & $\mathrm{BIO}$ & 135 & 5 & nd & 27 \\
\hline & CHEM 60 & 124 & 16 & 0.4 & 20 \\
\hline & CHEM & 72 & nd & nd & 15 \\
\hline & 110 & & & & \\
\hline & PHYS & 106 & nd & nd & 20 \\
\hline \multirow[t]{6}{*}{ Biochar+soil } & UNAGED & 146 & 31 & 1 & 30 \\
\hline & $\mathrm{BIO}$ & 134 & 36 & 0.3 & 31 \\
\hline & CHEM 60 & 133 & 20 & 0.1 & 24 \\
\hline & CHEM & 128 & 18 & 0.7 & 44 \\
\hline & 110 & & & & \\
\hline & PHYS & 137 & 16 & 1 & 28 \\
\hline \multirow[t]{6}{*}{ Soil } & UNAGED & 147 & 6 & nd & 28 \\
\hline & $\mathrm{BIO}$ & 125 & 10 & nd & 26 \\
\hline & CHEM 60 & 149 & 3 & 0.6 & 25 \\
\hline & CHEM & 127 & 3 & 2 & 32 \\
\hline & 110 & & & & \\
\hline & PHYS & 160 & 4 & 0.3 & 31 \\
\hline
\end{tabular}

